\title{
A Mixed-Method Service Evaluation of Health Information Exchange in England: Technology Acceptance and Barriers and Facilitators to Adoption
}

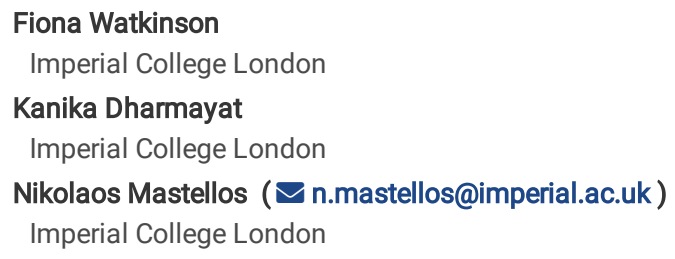

\section{Research Article}

Keywords: Health Information Exchange, Technology Acceptance, Technology Adoption, Unified Theory of Acceptance and Use of Technology, Normalisation Process Theory

Posted Date: April 16th, 2021

DOI: https://doi.org/10.21203/rs.3.rs-401470/v1

License: (c) (7) This work is licensed under a Creative Commons Attribution 4.0 International License. Read Full License

Version of Record: A version of this preprint was published at BMC Health Services Research on July 25th, 2021. See the published version at https://doi.org/10.1186/s12913-021-06771-z. 


\section{Abstract}

Background: The need for information exchange and integrated care has stimulated the development of interoperability solutions that bring together patient data across the health and care system to enable effective information sharing. Health Information Exchange (HIE) solutions have been shown to be effective in supporting patient care, however, user adoption often varies among users and care settings. This service evaluation aimed to measure user acceptance of HIE and explore barriers and facilitators to its wider uptake.

Methods: A mixed-method study design was used. A questionnaire was developed using the Unified Theory of Acceptance and Use of Technology and administered to HIE users to assess technology acceptance. Pearson $\mathrm{Chi}^{2}$ tests were used to examine differences in acceptance between user groups and care settings. Web-based, semi-structured interviews were conducted drawing on the Normalisation Process Theory to explore barriers and facilitators to adoption. Interview data were analysed thematically using the Framework Approach.

Results: A total of 105 HIE users completed the survey and another 12 participated in the interviews. Significant differences were found in HIE acceptance between users groups and care settings, with high adopters demonstrating higher acceptance and social care users showing lower acceptance. Participants identified several drivers to adoption, including increased information accessibility, better care coordination, informed decision-making, improved patient care, reduced duplication of procedures, and time and cost savings. However, they also highlighted a number of barriers, such as lack of awareness about the solution and its value, suboptimal communication strategies, inadequate training and lack of resources for knowledge dissemination, absence of champions to support the implementation, lack of end user involvement in the implementation and evaluation of HIE, unclear accountability and responsibility for the overall success of the programme, and patient confidentiality concerns.

Conclusions: Working to better engage stakeholders, considering the needs of users from different care settings, providing users with training resources and support to increase their knowledge and confidence in using the system, developing implementation strategies to seek user feedback and monitor performance, and using communication strategies to increase awareness of the product and its value, can help improve uptake and adoption of HIE.

\section{Background}

The increasing need for health and care information sharing and the availability of standards for secure data exchange across multiple Electronic Health Records (EHR) systems has led to the development of new solutions that bring together health and social care information from siloed sources to support person-centred care. Health Information Exchange (HIE) involves electronic mobilisation of patient information within and across organisations and between various EHR systems according to locally and/or nationally recognised standards to enable informed decision-making and improve the quality of healthcare in a population (1). HIE has been shown to provide a variety of benefits to health care systems, such as minimising gaps in patient histories, avoiding unnecessary duplication of procedures and investigations, reducing hospital admissions, referrals, and costs, and improving immunisation rates, patient safety, experience and outcomes (2-6).

These improvements are particularly important considering the shift to more integrated care models that require multiple providers in a local area to work together to achieve greater integration of health and social care services and improve population health. HIE has been shown to be particularly effective in connecting social care services, such as hospice care centres, which typically exist as siloed systems separate to the National Health Service (NHS). For example, the implementation of HIE in St. Josephs Hospice in Hackney, England, allowed the hospice to access over 100,000 records from 12 East London health and care organisations through the East London Patient Record (eLPR), while enabling eLPR to gain access to personalised urgent care plans designed through St. Josephs Hospice (7).

Despite the established benefits of HIE, user acceptance remains a prominent barrier in the uptake of HIE systems (8). While small-scale studies of HIE acceptance have shown positive results, currently, no studies have assessed user acceptance across a variety of contexts within England to account for the diverse needs that exist within different health and care settings and regions. This study assesses user acceptance of HIE across England and explores barriers and facilitators to its wider implementation. Capturing the perspectives of HIE users is hugely powerful as it demonstrates its value to their peers and patients and identifies barricades to adoption as well as opportunities for improvement.

\section{Methods}

\section{Study aim}

This study was designed to measure user acceptance of HIE across 15 NHS Trusts in England and explore barriers and facilitators to its wider uptake. At the time of the study (June-July 2020), HIE adoption varied amongst users and care settings in the participating HIE sites. It was therefore deemed important to generate insights into the observed differences in HIE uptake by analysing the relationship between user acceptance, contextual factors, and adoption.

\section{Study design}

A mixed-method study design was adopted to address the study aims, consisting of development and administration of an end-user survey to quantify HIE acceptance and web-based interviews to generate qualitative data surrounding perceived barriers and facilitators to uptake.

\section{Participants and setting}

Participants were users and implementation teams of Cerner's HIE platform from 15 NHS Trusts in England. Cerner's HIE is a cloud-based, vendor-agnostic solution enabling information sharing across acute, primary, community, social care, and mental health settings. Users can access HIE as a 'click-through' 
from their EHR system and get real-time access to patient data from disperse sources. When this study was conducted, HIE had been implemented across 15 NHS Trusts in England covering a population of $\approx 13$ million patients (Fig. 1).

\section{Data collection}

The HIE questionnaire was designed to collect demographic data, such as age, gender and occupation, and assess users' perceptions of HIE through a fivepoint Likert scale (ranging from 'strongly disagree' to 'strongly agree') designed using constructs from the Unified Theory of Acceptance and Use of Technology (UTAUT) (9). Following development, a link to the online survey, which was developed using Imperial College London's Qualtrics ${ }^{\circledR}$ survey tool, was sent to the participating HIE organisations who were responsible for administering the survey to their staff to protect participants' anonymity. The survey was designed to take less than 10 minutes to complete. The questionnaire remained open for 4 weeks, from mid-June 2020 to mid-July 2020 , with reminder emails to complete the survey sent weekly following initial questionnaire distribution.

An opt-in within the HIE questionnaire allowed participants to volunteer for a follow-up virtual interview exploring barriers and facilitators to the use of HIE by providing their email address. The research team reached out via email to provide participants with a sign-up link for an interview time. All participants were provided with a participant information sheet to brief them on stipulations of the study. Interview questions and format were designed using the Normalisation Process Theory (NPT), which assesses four constructs: Coherence, Cognitive Preparation, Collective Action, and Reflexive Monitoring (10). While the NPT provided a framework for the interview structure, general questions on overall perception of barriers and facilitators to the implementation of HIE within health systems were also introduced. Interviews were semi-structured and conducted using Microsoft Teams virtual conferencing software and lasted between 15 and 30 minutes. Interviews were recorded with permission of the interviewee to ensure each interview could be accurately transcribed later for analysis. Recordings were deleted following transcription. The transcriptions are stored securely in servers at Imperial College London.

\section{Data analysis}

Survey data were exported from the Imperial College London Qualtricsß system, cleaned, coded and analysed using Stata ${ }^{\circledR}$ Version 15 . Due to the small sample size, all five-point Likert scales were condensed to three levels (i.e. 'Disagree', consisting of 'Strongly Disagree' and 'Disagree'; 'Neither Agree nor Disagree'; and 'Agree', consisting of 'Agree' and 'Strongly Agree') to achieve a clearer delineate the direction of responses amongst user groups (i.e. low, medium and high level users). The groups were defined by Cerner Corporation following analysis of monthly HIE usage data from the participating sites. Descriptive statistics and Pearson's $\mathrm{Chi}^{2}$ tests with a significance level of $\mathrm{p} \leq .05$ were used to assess differences between user groups by demographic characteristics and HIE acceptance.

Interview data were transcribed verbatim and analysed thematically using the Framework Approach (11). This involved familiarisation with the data through reading the interview transcripts, identification of a priori and emerging themes using the notes from the transcripts, indexing of relevant portions of interview data to corresponding thematic categories, charting, mapping and interpretation by summarising priori and emergent themes using the charted themes and relevant quotations from the transcripts.

\section{Results}

\section{Demographics}

A total of $115 \mathrm{HIE}$ users completed the survey. Of those, 10 were excluded as they only completed the demographic portion of the survey. The demographics of the 105 respondents are shown in Table 1. The majority were high-level users (using HIE $5+$ times per week), followed by mid-level (3-4 times per week) and low-level (1-2 times per week) users. There were no significant differences between user groups across all demographic variables. 
Table 1

Survey demographics

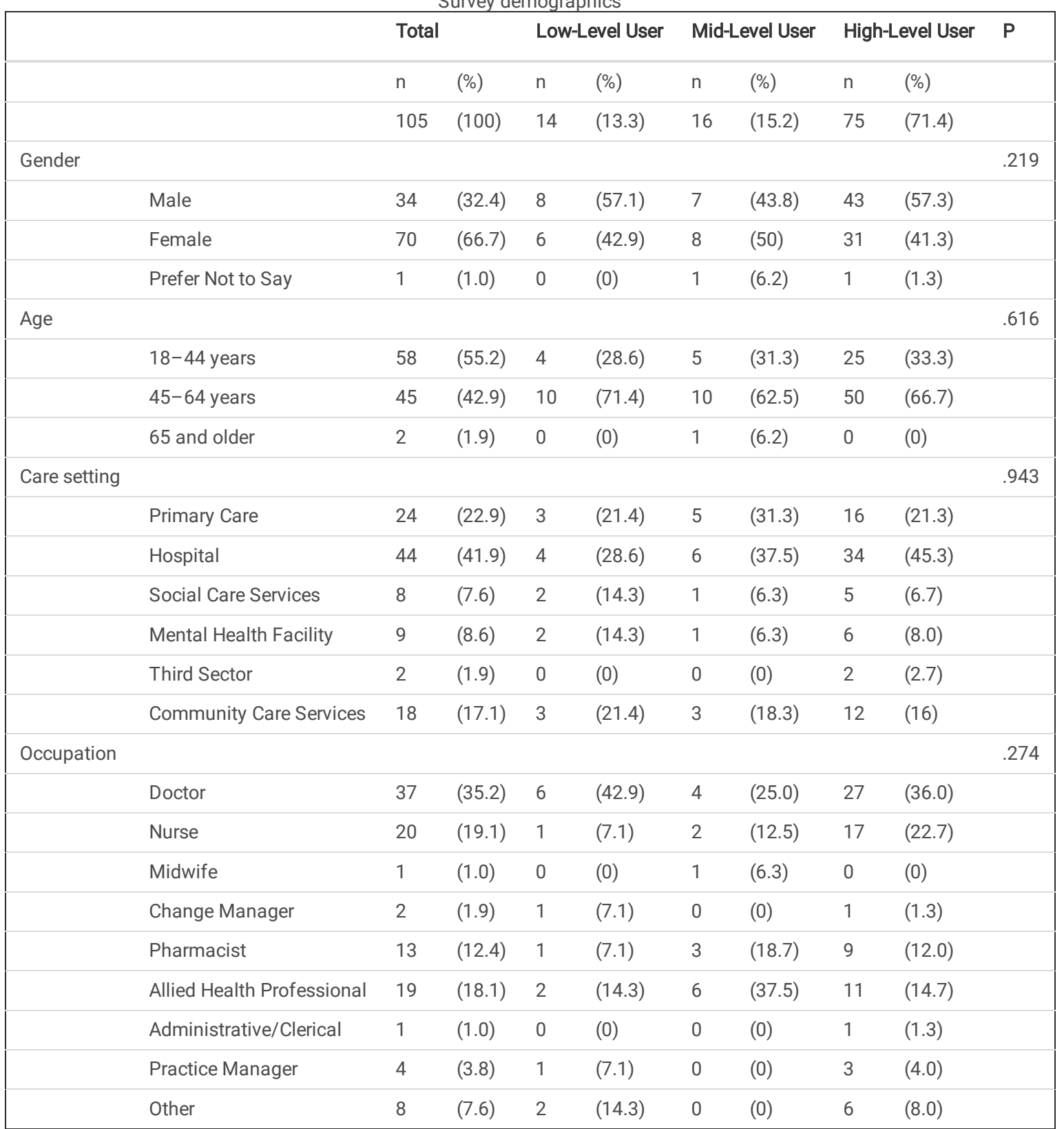

Twelve individuals participated in an interview. Most were females $(n=9)$ and represented the $45-65$ year-old category $(n=7)$, with the rest being between $18-$ 44 years old. Three participants were members of the implementation team, while the rest were end users. Most interviewees $(n=7)$ were from an acute setting with representation from four more settings: mental health $(n=2)$, primary care $(n=1)$, community care $(n=1)$ and social care $(n=1)$. Most participants were doctors $(n=4)$, followed by allied health professionals $(n=3)$, nurses $(n=2)$, a pharmacist, a social worker, and a change manager.

\section{HIE acceptance}

Overall, technology acceptance (Table 2) was high with most users finding HIE useful (PU), easy to use (PEOU), relevant to their role (REL) and thus using it routinely $(\mathrm{HT})$. In addition, participants showed a clear intention to use HIE regularly in the future, with some users also being keen to increase its use (BI). Enjoyment responses were mixed with $45 \%$ of users finding HIE enjoyable to use and $40 \%$ providing a neutral response. However, respondents felt that there was no push from the top or other HIE advocates to use HIE (SN). Also, the training provided was inadequate and there was no support available when users needed support with HIE (FC). 


\begin{tabular}{|c|c|c|c|c|}
\hline Construct & Survey Question & Disagree & Neutral & Agree \\
\hline & & $\mathrm{n}(\%)$ & $\mathrm{n}(\%)$ & $\mathrm{n}(\%)$ \\
\hline PU1 & HIE has saved me time at work & $4(3.8)$ & $11(10.5)$ & $90(85.7)$ \\
\hline PU2 & HIE has made my job easier & $2(1.9)$ & $7(6.7)$ & $96(91.4)$ \\
\hline PU3 & Using HIE helps me to be a better healthcare provider & $2(1.9)$ & $14(13.3)$ & $89(84.8)$ \\
\hline PU4 & Using HIE supports critical aspects of my patients' healthcare & $3(2.9)$ & $6(5.7)$ & $96(91.4)$ \\
\hline PU5 & Using HIE enhances my effectiveness as a healthcare provider & $2(1.9)$ & $12(11.4)$ & $91(86.7)$ \\
\hline PU6 & Overall, HIE is useful to me in managing my patients' healthcare & $1(1.0)$ & $8(7.6)$ & $96(91.4)$ \\
\hline PEOU1 & Learning how to use HIE was easy for me & $5(4.8)$ & $11(10.5)$ & $89(84.7)$ \\
\hline PEOU2 & It was easy for me to become skilful at using HIE & $9(8.6)$ & $10(9.5)$ & $86(81.9)$ \\
\hline PEOU3 & I find HIE easy to use & $9(8.6)$ & $12(11.4)$ & $84(80.0)$ \\
\hline PE1 & $\mathrm{HIE}$ is enjoyable to use & $16(15.2)$ & $42(40.0)$ & $47(44.8)$ \\
\hline REL1 & In my job, using HIE is important & $1(1.0)$ & $1(1.0)$ & $103(98.0)$ \\
\hline REL2 & The use of HIE is pertinent to many of my job-related tasks & $2(1.9)$ & $10(9.5)$ & $93(88.6)$ \\
\hline HT1 & The use of HIE has become a habit for me & $6(5.7)$ & $13(12.4)$ & $86(81.9)$ \\
\hline $\mathrm{Bl} 1$ & I intend to use HIE regularly & $0(0)$ & $4(3.8)$ & $101(96.2)$ \\
\hline $\mathrm{B} 12$ & I intend to increase the amount I use HIE in the future & $0(0)$ & $33(31.4)$ & $72(68.6)$ \\
\hline SN1 & People in my workplace promote the use of HIE & $13(12.4)$ & $24(22.9)$ & $68(64.7)$ \\
\hline SN2 & People who influence my behaviour at work use HIE & $8(7.6)$ & $31(29.5)$ & $66(62.9)$ \\
\hline SN3 & Most individuals in my workplace use HIE & $9(8.6)$ & $31(29.5)$ & $65(61.9)$ \\
\hline FC1 & I received adequate training when I began using HIE & $53(50.5)$ & $22(20.9)$ & $30(28.6)$ \\
\hline FC2 & I have the resources necessary to use HIE & $7(6.7)$ & $16(15.2)$ & $82(78.1)$ \\
\hline FC3 & I have the knowledge necessary to use HIE & $4(3.8)$ & $15(14.3)$ & $86(81.9)$ \\
\hline FC4 & HIE is compatible with the other electronic health systems I use at work & $16(15.2)$ & $25(23.8)$ & $64(61.0)$ \\
\hline FC5 & I can receive help when I have difficulties using HIE & $39(37.1)$ & $37(35.2)$ & $29(27.7)$ \\
\hline
\end{tabular}

Table 3 shows the breakdown of responses by user group. High adopters provided consistently more positive responses compared to low- and medium-level users. Significant differences were observed between user groups in terms of how relevant HIE was in their job (REL2), whether using HIE had become a habit for them (HT1), the impact of individuals who influence their behaviour on HIE adoption (SN1, SN2), and the possession of the necessary knowledge to use HIE (FC3). 


\begin{tabular}{|c|c|c|c|c|c|c|c|c|c|c|c|}
\hline \multirow[b]{2}{*}{ Construct } & \multicolumn{3}{|c|}{ Low-level users } & \multicolumn{3}{|c|}{ Medium-level users } & \multicolumn{2}{|c|}{ High-level users } & \multirow[b]{2}{*}{ Agree } & \multirow[b]{2}{*}{$x^{2}$} & \multirow[b]{2}{*}{$P$} \\
\hline & Disagree & Neutral & Agree & Disagree & Neutral & Agree & Disagree & Neutral & & & \\
\hline & $\mathrm{n}(\%)$ & $\mathrm{n}(\%)$ & $\mathrm{n}(\%)$ & $\mathrm{n}(\%)$ & $\mathrm{n}(\%)$ & n (\%) & $\mathrm{n}(\%)$ & $\mathrm{n}(\%)$ & $\mathrm{n}(\%)$ & $(4,105)$ & \\
\hline PU1 & $2(14.3)$ & $1(7.1)$ & $11(78.6)$ & $1(6.3)$ & $2(12.5)$ & $13(81.3)$ & $1(1.3)$ & $8(10.7)$ & $66(88)$ & 5.87 & .209 \\
\hline PU2 & $1(7.1)$ & $2(14.3)$ & $11(78.6)$ & $0(0)$ & $2(12.5)$ & $14(87.5)$ & $1(1.3)$ & $3(4)$ & $71(94.7)$ & 5.65 & .227 \\
\hline PU3 & $0(0)$ & $3(21.4)$ & $11(78.6)$ & $1(6.3)$ & $4(25)$ & $11(68.7)$ & $1(1.3)$ & $7(9.3)$ & $67(89.3)$ & 5.93 & .204 \\
\hline PU4 & $0(0)$ & $1(7.1)$ & $13(92.9)$ & $1(6.3)$ & $2(12.5)$ & $13(81.2)$ & $2(2.7)$ & $3(4)$ & 70 (93.3) & 2.99 & .559 \\
\hline PU5 & $0(0)$ & $2(14.3)$ & $12(85.7)$ & $0(0)$ & $5(31.3)$ & $11(68.7)$ & $2(2.7)$ & $5(11.7)$ & $68(90.6)$ & 8.62 & .071 \\
\hline PU6 & $0(0)$ & $1(7.1)$ & 13 (92.) & $0(0)$ & $3(13.8)$ & $13(81.2)$ & $1(1.3)$ & $4(5.3)$ & 70 (91.4) & 3.73 & .443 \\
\hline PEOU1 & $1(7.1)$ & $1(7.1)$ & 12 (85.7) & $1(6.3)$ & $4(25)$ & $11(68.7)$ & $3(4)$ & $6(8)$ & $66(88)$ & 4.72 & .317 \\
\hline \multirow[t]{2}{*}{ PEOU2 } & $1(7.1)$ & & & $3(18.8)$ & & & & & & & .051 \\
\hline & & $0(0)$ & 13 (92.9) & & $4(25)$ & $9(56.2)$ & $5(6.7)$ & $6(8)$ & 64 (85.3) & 9.42 & \\
\hline PEOU3 & $1(7.1)$ & $1(7.1)$ & $12(85.7)$ & $1(6.3)$ & $5(31.2)$ & $10(62.5)$ & $7(9.3)$ & $6(8)$ & $62(82.7)$ & 7.43 & .115 \\
\hline PE1 & 2 (14.3) & $7(50)$ & $5(35.7)$ & $2(12.5)$ & $8(50)$ & $6(37.5)$ & $12(16)$ & $27(36)$ & $36(48)$ & 1.79 & .775 \\
\hline REL1 & $1(7.1)$ & $0(0)$ & 13 (92.9) & $0(0)$ & $0(0)$ & $16(100)$ & $0(0)$ & $1(1.3)$ & 74 (98.7) & 6.95 & .139 \\
\hline REL2 & $1(7.1)$ & $2(14.3)$ & $11(78.6)$ & $0(0)$ & 5 (31.3) & $11(68.7)$ & $1(1.3)$ & $3(4)$ & 71 (94.7) & 14.3 & .006 \\
\hline HT1 & $3(21.4)$ & $3(21.4)$ & $8(57.1)$ & $3(18.8)$ & 3 (18.8) & $10(62.5)$ & $0(0)$ & $7(9.3)$ & 68 (90.7) & 19.59 & .001 \\
\hline $\mathrm{Bl} 1$ & $0(0)$ & $1(7.1)$ & $13(92.9)$ & $0(0)$ & 2 (12.5) & $14(87.5)$ & $0(0)$ & $1(1.3)$ & 74 (98.7) & 4.98 & .083 \\
\hline $\mathrm{Bl} 2$ & $0(0)$ & $6(42.9)$ & 8 (57.1) & $0(0)$ & $6(37.5)$ & $10(62.5)$ & $0(0)$ & $21(28)$ & $54(72)$ & 1.53 & .465 \\
\hline SN1 & 5 (35.7) & $4(28.6)$ & $5(35.7)$ & $1(6.3)$ & $3(18.8)$ & $12(75)$ & $7(9.3)$ & $17(22.7)$ & $51(68)$ & 9.73 & .045 \\
\hline SN2 & 3 (21.4) & $6(42.9)$ & $5(35.7)$ & $1(6.3)$ & 7 (43.8) & $8(50)$ & $4(5.3)$ & $18(24)$ & $53(70.7)$ & 9.56 & .048 \\
\hline SN3 & $0(0)$ & $6(42.9)$ & 8 (57.1) & $2(12.5)$ & 3 (18.8) & $11(68.8)$ & $7(9.3)$ & $22(29.3)$ & $46(61.3)$ & 3.19 & .527 \\
\hline $\mathrm{FC} 1$ & 8 (57.1) & 3 (21.4) & 3 (21.4) & $7(43.8)$ & $4(25)$ & 5 (31.3) & 38 (50.7) & $15(20)$ & $22(29.3)$ & 0.73 & .947 \\
\hline $\mathrm{FC} 2$ & $1(7.1)$ & $2(14.3)$ & $11(78.6)$ & $1(6.3)$ & 3 (18.7) & $12(75)$ & $5(6.7)$ & $11(14.7)$ & $59(78.6)$ & 0.19 & .996 \\
\hline FC3 & $1(7.1)$ & $4(28.57)$ & 9 (64.5) & $1(6.3)$ & 5 (31.3) & $10(62.5)$ & $2(2.7)$ & $6(8)$ & 67 (89.3) & 9.98 & .041 \\
\hline $\mathrm{FC} 4$ & $1(7.1)$ & $4(28.6)$ & 9 (64.3) & $4(25)$ & 3 (18.8) & $9(56.2)$ & $11(14.7)$ & $18(24)$ & $46(61.3)$ & 2.01 & .734 \\
\hline FC5 & $4(28.6)$ & 6 (42.9) & $4(28.6)$ & 3 (18.6) & $8(50)$ & 5 (31.3) & $32(42.7)$ & $23(35.7)$ & $20(26.7)$ & 4.12 & .390 \\
\hline
\end{tabular}

When collapsing the low- and medium-user groups into one category and comparing with the high adopters category, additional significant differences were revealed (Table 4). High adopters were more likely to believe that using HIE enhances their effectiveness in their job (PU5) and intended to use HIE regularly in the future (BI1).

Table 4

HIE acceptance between user groups

\begin{tabular}{|llllllllll|}
\hline \multicolumn{7}{|c|}{ Low/medium-level users } & High-level users \\
Construct & Disagree & Neutral & Agree & Disagree & Neutral & Agree & $\mathbf{x}^{2}$ & P \\
& $\mathrm{n}(\%)$ & $\mathrm{n}(\%)$ & $\mathrm{n}(\%)$ & $\mathrm{n}(\%)$ & $\mathrm{n}(\%)$ & $\mathrm{n}(\%)$ & $(4,105)$ & \\
\hline PU5 & $0(0)$ & $7(23.3)$ & $23(76.7)$ & $2(2.7)$ & $5(6.7)$ & $68(90.7)$ & 6.49 & .039 \\
\hline REL2 & $0(0)$ & $5(31.3)$ & $11(68.7)$ & $1(1.3)$ & $3(4.0)$ & $71(94.7)$ & 14.3 & .006 \\
HT1 & $3(18.8)$ & $3(18.8)$ & $10(62.5)$ & $0(0)$ & $7(9.3)$ & $68(90.7)$ & 19.59 & .001 \\
\hline BI1 & $0(0)$ & $3(10)$ & $27(90)$ & $0(0)$ & $1(1.3)$ & $74(98.7)$ & 4.4 & .036 \\
\hline SN2 & $4(13.3)$ & $13(43.3)$ & $13(43.3)$ & $4(5.3)$ & $18(24)$ & $53(70.7)$ & 7.06 & .029 \\
\hline FC3 & $1(6.3)$ & $5(31.3)$ & $10(62.5)$ & $2(2.7)$ & $6(8)$ & $67(89.3)$ & 9.98 & .041 \\
\hline
\end{tabular}

Significant differences were also found between care settings (Table 5). The perceived usefulness category (PU4, PU5, PU6) showed notable differences, with only $62.5 \%$ of social care respondents agreeing that HIE was helpful for managing patient care as compared to $91.7 \%$ in primary care, $100 \%$ in hospitals, and 
$88.9 \%$ in mental health and community care. Similar rates were seen in enhancing effectiveness as a provider and supporting critical aspects of healthcare, with only $37.5 \%$ and $75 \%$ of social services agreeing with these statements, respectively. A significant difference was also noted in the subjective norm category (SN3), with only $12.5 \%$ of social care and $50 \%$ of community care users indicating HIE was used widely in their workplace. Finally, respondents had significantly different opinions with regards to two facilitating conditions (FC2, FC5), with half of social care respondents indicating that they had the resources to use $\mathrm{HIE}$, and all third sector users saying that they could receive help with HIE.

\begin{tabular}{|c|c|c|c|c|c|c|c|c|c|c|c|c|c|}
\hline \multirow[b]{2}{*}{ Question } & \multicolumn{3}{|c|}{ Primary Care $(n=24)$} & \multicolumn{3}{|c|}{ Acute Care $(n=44)$} & \multicolumn{3}{|c|}{ Community Care $(n=18)$} & \multicolumn{3}{|c|}{ Mental Health $(n=9)$} & \multirow{2}{*}{$\begin{array}{l}\text { Social Car } \\
\text { Disagree }\end{array}$} \\
\hline & Disagree & Neutral & Agree & Disagree & Neutral & Agree & Disagree & Neutral & Agree & Disagree & Neutral & Agree & \\
\hline & n (\%) & n (\%) & n (\%) & $\mathrm{n}(\%)$ & $\mathrm{n}(\%)$ & $\mathrm{n}(\%)$ & $\mathrm{n}(\%)$ & $\mathrm{n}(\%)$ & $\mathrm{n}(\%)$ & $\mathrm{n}(\%)$ & $\mathrm{n}(\%)$ & $\mathrm{n}(\%)$ & $\mathrm{n}(\%)$ \\
\hline PU1 & $1(4.2)$ & $\begin{array}{l}4 \\
(16.7)\end{array}$ & $\begin{array}{l}19 \\
(79.2)\end{array}$ & $2(4.5)$ & $4(9.1)$ & $\begin{array}{l}38 \\
(86.4)\end{array}$ & $0(0)$ & $\begin{array}{l}2 \\
(11.1)\end{array}$ & $\begin{array}{l}16 \\
(88.9)\end{array}$ & $1(11.1)$ & $\begin{array}{l}1 \\
(11.1)\end{array}$ & $\begin{array}{l}7 \\
(77.8)\end{array}$ & $0(0)$ \\
\hline PU2 & $0(0)$ & $\begin{array}{l}3 \\
(12.5)\end{array}$ & $\begin{array}{l}21 \\
(87.5)\end{array}$ & $1(2.3)$ & $1(2.3)$ & $\begin{array}{l}42 \\
(95.4)\end{array}$ & $0(0)$ & $\begin{array}{l}2 \\
(11.1)\end{array}$ & $\begin{array}{l}16 \\
(88.9)\end{array}$ & $1(11.1)$ & $\begin{array}{l}1 \\
(11.1)\end{array}$ & $\begin{array}{l}7 \\
(77.8)\end{array}$ & $0(0)$ \\
\hline PU3 & $1(4.2)$ & $\begin{array}{l}3 \\
(12.5)\end{array}$ & $\begin{array}{l}20 \\
(83.3)\end{array}$ & $0(0)$ & $3(6.8)$ & $\begin{array}{l}41 \\
(93.2)\end{array}$ & $0(0)$ & $\begin{array}{l}3 \\
(16.7)\end{array}$ & $\begin{array}{l}15 \\
(83.3)\end{array}$ & $0(0)$ & $\begin{array}{l}1 \\
(11.1)\end{array}$ & $\begin{array}{l}8 \\
(88.9)\end{array}$ & $1(12.5)$ \\
\hline PU4 & $0(0)$ & $2(8.3)$ & $\begin{array}{l}22 \\
(91.7)\end{array}$ & $0(0)$ & $0(0)$ & $\begin{array}{l}44 \\
(100.0)\end{array}$ & $2(11.1)$ & $1(5.6)$ & $\begin{array}{l}15 \\
(83.3)\end{array}$ & $1(11.1)$ & $0(0)$ & $\begin{array}{l}8 \\
(88.9)\end{array}$ & $0(0)$ \\
\hline PU5 & $0(0)$ & $\begin{array}{l}3 \\
(12.5)\end{array}$ & $\begin{array}{l}21 \\
(87.5)\end{array}$ & $0(0)$ & $2(4.5)$ & $\begin{array}{l}42 \\
(95.5)\end{array}$ & $0(0)$ & $\begin{array}{l}2 \\
(11.1)\end{array}$ & $\begin{array}{l}16 \\
(88.9)\end{array}$ & $1(11.1)$ & $0(0)$ & $\begin{array}{l}8 \\
(88.9)\end{array}$ & $1(12.5)$ \\
\hline PU6 & $0(0)$ & $2(8.3)$ & $\begin{array}{l}22 \\
(91.7)\end{array}$ & $0(0)$ & $0(0)$ & $\begin{array}{l}44 \\
(100.0)\end{array}$ & $0(0)$ & $\begin{array}{l}2 \\
(11.1)\end{array}$ & $\begin{array}{l}16 \\
(88.9)\end{array}$ & $1(11.1)$ & $0(0)$ & $\begin{array}{l}8 \\
(88.9)\end{array}$ & $0(0)$ \\
\hline PEOU1 & $2(8.3)$ & $\begin{array}{l}4 \\
(16.7)\end{array}$ & $\begin{array}{l}18 \\
(75.0)\end{array}$ & $1(2.3)$ & $\begin{array}{l}5 \\
(11.4)\end{array}$ & $\begin{array}{l}38 \\
(86.4)\end{array}$ & $1(5.6)$ & $1(5.6)$ & $\begin{array}{l}16 \\
(88.9)\end{array}$ & $0(0)$ & $\begin{array}{l}1 \\
(11.1)\end{array}$ & $\begin{array}{l}8 \\
(88.9)\end{array}$ & $1(12.5)$ \\
\hline PEOU2 & $4(16.7)$ & $\begin{array}{l}4 \\
(16.7)\end{array}$ & $\begin{array}{l}16 \\
(66.7)\end{array}$ & $2(4.6)$ & $3(6.8)$ & $\begin{array}{l}39 \\
(88.6)\end{array}$ & $2(11.1)$ & $1(5,6)$ & $\begin{array}{l}15 \\
(83.3)\end{array}$ & $0(0)$ & $\begin{array}{l}2 \\
(22.2)\end{array}$ & $\begin{array}{l}7 \\
(77.8)\end{array}$ & $1(12.5)$ \\
\hline PEOU3 & $3(12.5)$ & $\begin{array}{l}5 \\
(20.8)\end{array}$ & $\begin{array}{l}16 \\
(66.7)\end{array}$ & $3(6.8)$ & $4(9.1)$ & $\begin{array}{l}37 \\
(84.1)\end{array}$ & $0(0)$ & $\begin{array}{l}2 \\
(11.1)\end{array}$ & $\begin{array}{l}16 \\
(88.9)\end{array}$ & $1(11.1)$ & $\begin{array}{l}1 \\
(11.1)\end{array}$ & $\begin{array}{l}7 \\
(77.8)\end{array}$ & $2(25.0)$ \\
\hline PE1 & $6(25)$ & $\begin{array}{l}9 \\
(37.5)\end{array}$ & $\begin{array}{l}9 \\
(37.5)\end{array}$ & $4(9.1)$ & $\begin{array}{l}13 \\
(29.5)\end{array}$ & $\begin{array}{l}27 \\
(61.4)\end{array}$ & 3 (16.7) & $\begin{array}{l}8 \\
(44.4)\end{array}$ & $\begin{array}{l}7 \\
(38.9)\end{array}$ & $1(11.1)$ & $\begin{array}{l}7 \\
(77.8)\end{array}$ & $\begin{array}{l}1 \\
(11.1)\end{array}$ & $2(25.0)$ \\
\hline REL1 & $0(0)$ & $0(0)$ & $\begin{array}{l}24 \\
(100.0)\end{array}$ & $0(0)$ & $0(0)$ & $\begin{array}{l}44 \\
(100.0)\end{array}$ & $1(5.6)$ & $0(0)$ & $\begin{array}{l}17 \\
(94.4)\end{array}$ & $0(0)$ & $\begin{array}{l}1 \\
(11.1)\end{array}$ & $\begin{array}{l}8 \\
(88.9)\end{array}$ & $0(0)$ \\
\hline REL2 & $0(0)$ & $\begin{array}{l}6 \\
(25.0)\end{array}$ & $\begin{array}{l}18 \\
(75.0)\end{array}$ & $0(0)$ & $2(4.6)$ & $\begin{array}{l}42 \\
(95.4)\end{array}$ & $1(5.6)$ & $\begin{array}{l}2 \\
(11.1)\end{array}$ & $\begin{array}{l}15 \\
(83.3)\end{array}$ & $1(11.1)$ & $0(0)$ & $\begin{array}{l}8 \\
(88.9)\end{array}$ & $0(0)$ \\
\hline HT1 & $1(4.2)$ & $\begin{array}{l}4 \\
(16.7)\end{array}$ & $\begin{array}{l}19 \\
(79.2)\end{array}$ & $2(4.6)$ & $4(9.1)$ & $\begin{array}{l}38 \\
(86.4)\end{array}$ & $2(11.1)$ & $\begin{array}{l}4 \\
(22.2)\end{array}$ & $\begin{array}{l}12 \\
(66.7)\end{array}$ & $0(0)$ & $0(0)$ & $\begin{array}{l}9 \\
(100.0)\end{array}$ & $1(12.5)$ \\
\hline BI1 & $0(0)$ & $1(4.2)$ & $\begin{array}{l}23 \\
(95.8)\end{array}$ & $0(0)$ & 2 (4.6) & $\begin{array}{l}42 \\
(95.4)\end{array}$ & $0(0)$ & $1(5.6)$ & $\begin{array}{l}17 \\
(94.4)\end{array}$ & $0(0)$ & $0(0)$ & $\begin{array}{l}9 \\
(100.0)\end{array}$ & $0(0)$ \\
\hline $\mathrm{BI} 2$ & $0(0)$ & $\begin{array}{l}5 \\
(20.8)\end{array}$ & $\begin{array}{l}19 \\
(79.2)\end{array}$ & $0(0)$ & $\begin{array}{l}12 \\
(27.3)\end{array}$ & $\begin{array}{l}32 \\
(72.7)\end{array}$ & $0(0)$ & $\begin{array}{l}8 \\
(44.4)\end{array}$ & $\begin{array}{l}10 \\
(55.6)\end{array}$ & $0(0)$ & $\begin{array}{l}4 \\
(44.4)\end{array}$ & $\begin{array}{l}5 \\
(55.6)\end{array}$ & $0(0)$ \\
\hline SN1 & $3(12.5)$ & $\begin{array}{l}3 \\
(12.5)\end{array}$ & $\begin{array}{l}18 \\
(75.0)\end{array}$ & $5(11.4)$ & $\begin{array}{l}9 \\
(20.4)\end{array}$ & $\begin{array}{l}30 \\
(68.2)\end{array}$ & $2(11.1)$ & $\begin{array}{l}8 \\
(44.4)\end{array}$ & $\begin{array}{l}8 \\
(44.4)\end{array}$ & $2(22.2)$ & $\begin{array}{l}1 \\
(11.1)\end{array}$ & $\begin{array}{l}6 \\
(66.7)\end{array}$ & $1(12.5)$ \\
\hline SN2 & $3(12.5)$ & $6(25)$ & $\begin{array}{l}15 \\
(62.5)\end{array}$ & $1(2.3)$ & $\begin{array}{l}12 \\
(27.3)\end{array}$ & $\begin{array}{l}31 \\
(70.4)\end{array}$ & $1(5.6)$ & $\begin{array}{l}8 \\
(44.4)\end{array}$ & $\begin{array}{l}9 \\
(50.0)\end{array}$ & $1(11.1)$ & $\begin{array}{l}3 \\
(33.3)\end{array}$ & $\begin{array}{l}5 \\
(55.6)\end{array}$ & $2(25.0)$ \\
\hline SN3 & $3(12.5)$ & $\begin{array}{l}5 \\
(20.8)\end{array}$ & $\begin{array}{l}16 \\
(66.7)\end{array}$ & $1(2.3)$ & $\begin{array}{l}11 \\
(25.0)\end{array}$ & $\begin{array}{l}32 \\
(72.7)\end{array}$ & $1(5.6)$ & $\begin{array}{l}8 \\
(44.4)\end{array}$ & $\begin{array}{l}9 \\
(50.0)\end{array}$ & 3 (33.3) & $\begin{array}{l}1 \\
(11.1)\end{array}$ & $\begin{array}{l}5 \\
(55.6)\end{array}$ & $1(12.5)$ \\
\hline FC1 & $\begin{array}{l}13 \\
(54.2)\end{array}$ & $\begin{array}{l}6 \\
(25.0)\end{array}$ & $\begin{array}{l}5 \\
(20.8)\end{array}$ & $\begin{array}{l}20 \\
(45.5)\end{array}$ & $\begin{array}{l}8 \\
(18.2)\end{array}$ & $\begin{array}{l}16 \\
(36.4)\end{array}$ & $\begin{array}{l}11 \\
(61.1)\end{array}$ & $\begin{array}{l}5 \\
(27.8)\end{array}$ & $\begin{array}{l}2 \\
(11.1)\end{array}$ & $6(66.7)$ & $\begin{array}{l}1 \\
(11.1)\end{array}$ & $\begin{array}{l}2 \\
(22.2)\end{array}$ & $3(37.5)$ \\
\hline $\mathrm{FC} 2$ & $2(8.3)$ & $\begin{array}{l}6 \\
(25.0)\end{array}$ & $\begin{array}{l}16 \\
(66.7)\end{array}$ & $0(0)$ & $3(6.8)$ & $\begin{array}{l}41 \\
(93.2)\end{array}$ & $2(11.1)$ & $\begin{array}{l}3 \\
(16.7)\end{array}$ & $\begin{array}{l}13 \\
(72.2)\end{array}$ & $0(0)$ & $\begin{array}{l}2 \\
(22.2)\end{array}$ & $\begin{array}{l}7 \\
(77.8)\end{array}$ & $2(25.0)$ \\
\hline FC3 & $2(8.3)$ & $\begin{array}{l}7 \\
(29.2)\end{array}$ & $\begin{array}{l}15 \\
(62.5)\end{array}$ & $1(2.3)$ & $3(6.8)$ & $\begin{array}{l}40 \\
(90.9)\end{array}$ & $0(0)$ & $\begin{array}{l}3 \\
(16.7)\end{array}$ & $\begin{array}{l}15 \\
(83.3)\end{array}$ & $0(0)$ & $\begin{array}{l}1 \\
(11.1)\end{array}$ & $\begin{array}{l}8 \\
(88.9)\end{array}$ & $1(12.5)$ \\
\hline FC4 & $7(29.2)$ & $\begin{array}{l}7 \\
(29.2)\end{array}$ & $\begin{array}{l}10 \\
(41.6)\end{array}$ & $3(6.8)$ & $\begin{array}{l}9 \\
(20.5)\end{array}$ & $\begin{array}{l}32 \\
(72.7)\end{array}$ & $3(16.7)$ & $\begin{array}{l}4 \\
(22.2)\end{array}$ & $\begin{array}{l}11 \\
(61.1)\end{array}$ & $1(11.1)$ & $\begin{array}{l}3 \\
(33.3)\end{array}$ & $\begin{array}{l}5 \\
(55.6)\end{array}$ & $1(12.5)$ \\
\hline FC5 & $\begin{array}{l}12 \\
(50.0)\end{array}$ & $\begin{array}{l}9 \\
(37.5)\end{array}$ & $\begin{array}{l}3 \\
(12.5)\end{array}$ & $\begin{array}{l}11 \\
(25.0)\end{array}$ & $\begin{array}{l}15 \\
(34.1)\end{array}$ & $\begin{array}{l}18 \\
(40.9)\end{array}$ & 7 (38.9) & $\begin{array}{l}9 \\
(50.0)\end{array}$ & $\begin{array}{l}2 \\
(11.1)\end{array}$ & $4(44.4)$ & $\begin{array}{l}4 \\
(44.4)\end{array}$ & $\begin{array}{l}1 \\
(11.1)\end{array}$ & $5(62.5)$ \\
\hline
\end{tabular}




\section{Barriers and facilitators to adoption}

Expectations of HIE varied across participants, with many not being aware of the solution at the time when HIE was implemented and therefore having no expectations. Stakeholder involvement during the implementation was relatively low with most participants stating that clinical teams and end-users were not consulted about the product prior to its implementation and that they were only made aware through an email

notifying them of the upcoming implementation. Following HIE deployment, end users said that they had not been contacted for feedback and indicated that they would like to provide feedback through some organised forum.

"I'm a senior social worker so l've got my own case load and supervise people with their own case-loads, and none of us at our level within my team were involved in that."

All participants agreed that end-users did not receive any training beyond email communications in a few cases. However, some also said that training on the system was not necessary. Participants suggested using super-users or champions to disseminate knowledge of the product, as well as reference materials such as demo videos and user manuals that use terminology which is appropriate for different regions and care settings. Others suggested regular reminders from Information Technology (IT) teams to help with learning the system and utilising it appropriately. Notably, most participants felt that a course or active training class would not be necessary for existing users, but HIE training on induction would be helpful.

It would be probably quite nice to be able to refer to something and say can I find this, could I use it for this, could I use it for that?

"I don't think sending me on a course would help me, I just need to practice it a bit more and be reminded."

End users felt that HIE use is down to individual practitioners with no accountability for uptake. However, implementation team participants stated that IT and change team members held their own teams accountable for diffusion and dispersed demo sessions and sent reminders to users to increase adoption. This was also acknowledged by some end users.

"Funnily enough, our IT teams reminded us a few times for those who hadn't used it so that was really good, that was quite clever."

All participants indicated that HIE was useful in their work as it allows real-time information accessibility within seconds, saving them time, enabling better decision-making, improving patient safety, increasing efficiency, and reducing duplication of procedures.

It's provided us a platform for innovative practice, better patients outcome, joining up systems which is so important, integrating different systems instead of working in isolation. Its saving us time so it translates into clinical efficiency as well.

However, some participants also felt that there is room for improvement, especially with increasing the area of HIE coverage and making the solution more user-friendly, particularly for social care and mental health users.

"Once you actually open the tab and go into HIE it doesn't look like anything we use in adult social care. "

Some participants recognised consent and privacy issues around patient confidentiality as a potential barrier. Others highlighted the importance of stakeholder engagement and improved communication strategies to increase awareness of the product and its value and thereby uptake. Communication was acknowledged as a key issue, with social care and mental health participants most consistently indicating a lack of awareness and suggesting better email communications.

What pains me is there not strategy for making sure that everybody knows what this is and how they can use it.

Nobody tells you about it, nobody values it particularly, it's not high on anybody's agenda.

"I suppose communication. Email, or having a Microsoft meeting, inviting end-user, multidisciplinary team members to show what is HIE about, informing teams what HIE means, what sort of information we can access."

The main drivers and barriers to adoption are listed below.

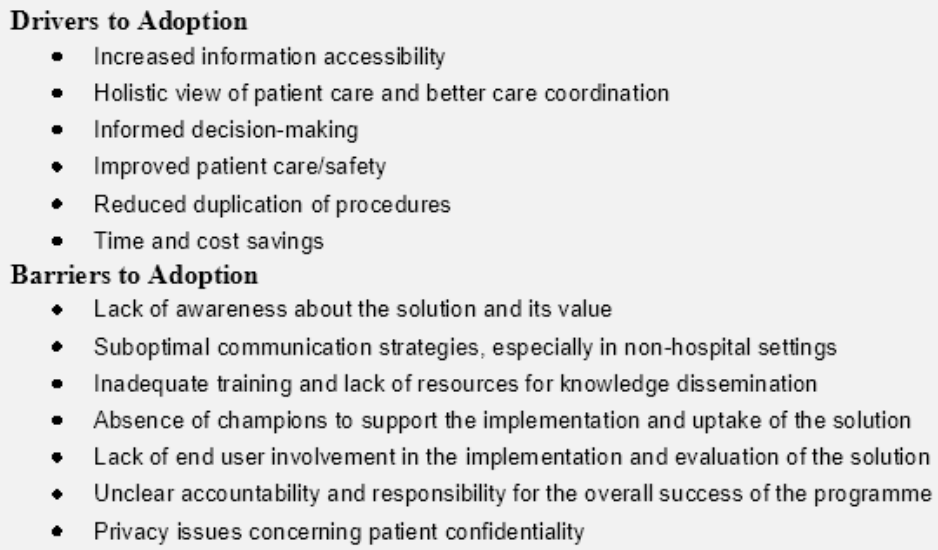




\section{Discussion}

The primary objective of the study was to measure user acceptance of HIE and analyse it by use level and setting through the administration of a survey utilising the UTAUT framework. The UTAUT has been frequently used across a variety of technologies and has shown to explain variance in use behaviour and intentions among end-users $(12,13)$. A secondary objective was to explore barriers and facilitators to HIE uptake using the NPT, which has been shown to be a valid and reliable method to assess implementation processes in a variety of contexts (14).

The findings show that most users perceived HIE useful, easy to use and relevant to their role. Most respondents showed a clear intention to use HIE in the future, with some also being keen to increase its use. However, the survey also revealed some gaps in the implementation strategy which may have impeded adoption. There were also significant differences in user acceptance between user groups, indicating an association between technology acceptance and use of technology.

Habitual use showed the largest statistically significant difference amongst user groups. Habit as a UTAUT construct has shown to be largely influenced by other factors such as importance to job, satisfaction, and experience with IT (15). Given this, the difference in relevance between user groups that was also identified may be feeding into the lack of habit development observed in lower-lever user groups. Habitual usage amongst end-users is important to establish as it has shown to increase the uptake and use of digital technology (16).

Interviewees also indicated that HIE use was not embedded into their practice and expressed the need for further awareness, reminders about the product, and tools for its use to improve adoption. HIE organisations should develop communication strategies to ensure that all end-users are aware of the product, see the value in using it, and have the resources they need to optimise its use in specific contexts.

Subjective norm was also significantly different across user groups, with lower-level users experiencing significantly lower subjective normalisation of the use of HIE among their peers than high-level users. Subjective norm relates to social proof theory, or the idea of individuals looking to others within their environment for confirmation of behaviour, which has been well-established and applied to a variety of contexts, including digital technology and healthcare $(17,18)$. The establishment of social proof is widely used as a marketing tool for companies and organisations, and should similarly be applied within HIE implementation strategy plans to increase acceptance and uptake (19). The use of HIE ambassadors could promote subjective normalisation of HIE and further increase user acceptance and adoption.

There was also agreement across all user groups that training was inadequate. Similarly, all interviewees indicated that they received little to no training and that, while classroom-style teaching was not necessary, in situ training videos or resource manuals on how to use the product would be beneficial. The need for appropriate digital literacy was also highlighted, with high-level users indicating they have the knowledge necessary to use HIE significantly more than lower-level users. It is therefore important to provide users from different health facilities with training and support to increase their knowledge and confidence in using HIE and improve their attitudes towards using the system $(20,21)$.

Social care services exhibited lower levels of perceived usefulness and social influence. Social and community care services also indicated issues with training and support. Training to use HIE was inadequate, and social care also noted they lacked the resources to properly use the system. The variations seen in user acceptance across care settings may be reflective of a lack of consideration of the profound differences between traditional and non-traditional care settings. Social and community care settings historically existed within silos separate from other NHS services; however, the demand for holistic care models has increased their integration with traditional NHS services (22). As HIE was primarily designed to be used in acute and primary care settings, considerations for services provided outside those settings were not made when designing the system. In fact, participants within social and community care services indicated issues with the user interface and with embedding the solution within their practices and noted that HIE did not look familiar to other systems used in those contexts. Therefore, it is important to tailor the design of HIE to those settings and provide users with resources and tools to learn how to use HIE effectively.

In line with findings from previous studies (3), use of HIE was found to result in time and cost savings, increased informed decision making, and collaborative care design. Several barriers were identified, including poor communication strategies and user awareness, inadequate training and resource provision, lack of stakeholder engagement, and privacy concerns. In developing implementation strategies, the consideration of all stakeholders must be addressed.

Participants indicated that end users were not involved pre- or post-implementation, and that system introduction was handled by IT and change teams. Engaging end users early in the process but also post-implementation could result in higher levels of system awareness, approval, and utilisation(23). As HIE expands, implementation strategies should be developed with collaborative end users in order to minimise several barriers identified within this study and increase uptake and adoption.

Although the survey was distributed to a diverse range of HIE users, gender was not equally represented in the survey sample, which can be due to the fact that $77 \%$ of the NHS workforce is female (24). In addition, participation to the survey was low due to the extreme pressures the NHS workforce was experiencing at the time of the study as a result of the COVID-19 pandemic. Also, most respondents were high adopters, which may have introduced some response bias due to the small representation of lower-level users in the study. Moreover, while the UTAUT framework was helpful for the design and understanding of the survey portion of the study, it may have oversimplified the relationships of the different constructs that feed into uptake and use of digital technology. Finally, the results of this study are not meant to be generalisable as service evaluations are primarily designed to assess current care standards and assist decisionmaking in particular settings, although they can be useful to others who are considering implementing a HIE solution.

Future studies should attempt to gain a larger sample size and generate findings that are more generalisable. Further investigation into the findings of this study surrounding inadequate training methods and limited support mechanisms for users would also be beneficial as this has shown to have a large impact on adoption and uptake of technologies (25). The development and testing of new implementation plans for HIE to identify the most appropriate methodology 
for application on a national scale should also be addressed. An analysis of the policies that currently exist regarding how data is shared on HIE may also be beneficial to understanding privacy concerns that may be a barrier to widespread use.

\section{Conclusions}

To the authors' knowledge, this is the first service evaluation of HIE acceptance across multiple NHS Trusts in England. The benefits of HIE were clear to endusers, however, there were also gaps in the implementation strategy, especially in non-traditional care settings. Working to better engage different stakeholders, considering the needs of end users from social and community care settings, creating adequate training plans and support users to increase their knowledge and confidence in using HIE, developing implementation strategies to seek user feedback and monitor performance, and using communication strategies to increase awareness of the product and its value, could help overcome the barriers identified in this study and achieve maximum benefits as HIE expands to novel settings.

\section{Abbreviations}

Bl: Behavioural Intention

EHR: Electronic Health Records

FC: Facilitating Conditions

GDPR: General Data Protection Regulation

HIE: Health Information Exchange

HT: Habit

IT: Information Technology

NHS: National Health Service

NPT: Normalisation Process Theory

PEOU: Perceived Ease Of Use

PU: Perceived Usefulness

REL: Relevance

SN: Subjective Norm

UTAUT: Unified Theory of Acceptance and Use of Technology

\section{Declarations}

\section{Ethics approval and consent to participate}

This study was conducted in accordance with the Declaration of Helsinki. Ethics committee review was not required as this study was conducted for the purpose of service evaluation, and service evaluations do not require ethics review according to Health Research Authority ("Defining Research. NRES guidance to help you decide if your project requires review by a Research Ethics Committee" (26)) and advice by the Imperial College Research Ethics Committee. To ensure compliance with general data protection regulation (GDPR), a privacy notice and participant information sheet were created with guidance from the Imperial College London data protection team. All participants provided informed consent through acceptance of the invitation to participate in the study.

\section{Consent for publication}

Not applicable.

\section{Availability of data and materials}

The datasets used and/or analysed during the current study are available from the corresponding author on reasonable request and with permission from the participating organisations.

\section{Competing interests}

FW and KD declare that they have no competing interests.

NM is a salaried employee at Cerner Corporation. 


\section{Funding}

Not applicable.

\section{Authors' contributions}

FW collected, analysed, and interpreted the study data and contributed to the writing of the manuscript. KD contributed to the design of the study and the writing of the manuscript. NM conceived and designed the study and was a major contributor in writing the manuscript. All authors read and approved the final manuscript.

\section{Acknowledgements}

We would like to thank all participants who gave up their time to participate in this study.

We would also like to thank the Cerner HIE team for facilitating the coordination of the study by administering the questionnaire to the participating HIE organisations.

Also, special thanks to the Imperial College Research Ethics Committee for reviewing the study protocol and the Data Protection team of the School of Public Health for their feedback on the participant information sheet and the survey and interview questionnaires.

\section{References}

1. Akhlaq A, Sheikh A, Pagliari C. Defining health information exchange: scoping review of published definitions. BMJ Health \&amp;amp; Care Informatics. 2016;23(4):684.

2. Bailey JE, Pope RA, Elliott EC, Wan JY, Waters TM, Frisse ME. Health information exchange reduces repeated diagnostic imaging for back pain. Ann Emerg Med. 2013;62(1):16-24.

3. Menachemi N, Rahurkar S, Harle CA, Vest JR. The benefits of health information exchange: an updated systematic review. J Am Med Inform Assoc. 2018;25(9):1259-65.

4. Rudin RS, Motala A, Goldzweig CL, Shekelle PG. Usage and effect of health information exchange: a systematic review. Ann Intern Med. 2014;161(11):803-11.

5. Shapiro JS, Mostashari F, Hripcsak G, Soulakis N, Kuperman G. Using health information exchange to improve public health. Am J Public Health. 2011;101(4):616-23.

6. Stockwell MS, Natarajan K, Ramakrishnan R, Holleran S, Forney K, Aponte A, et al. Immunization Data Exchange With Electronic Health Records. Pediatrics. 2016;137(6).

7. Edward S. P-100 Evaluation of the use of health information exchange at st joseph's hospice, london. BMJ Supportive \&amp; Palliative Care. 2019;9(Suppl 4):A46-A7.

8. Eden KB, Totten AM, Kassakian SZ, Gorman PN, McDonagh MS, Devine B, et al. Barriers and facilitators to exchanging health information: a systematic review. Int J Med Inform. 2016;88:44-51.

9. Venkatesh V, Morris MG, Davis GB, Davis FD. User Acceptance of Information Technology: Toward a Unified View. MIS Quarterly. 2003;27(3):425-78.

10. May CR, Cummings A, Girling M, Bracher M, Mair FS, May CM, et al. Using Normalization Process Theory in feasibility studies and process evaluations of complex healthcare interventions: a systematic review. Implementation Science. 2018;13(1):80.

11. Ritchie J, Lewis J, Nicholls CM, Ormston R. Qualitative research practice: A guide for social science students and researchers: sage; 2013.

12. Dwivedi YK, Rana NP, Chen H, Williams MD, editors. A Meta-analysis of the Unified Theory of Acceptance and Use of Technology (UTAUT)2011; Berlin, Heidelberg: Springer Berlin Heidelberg.

13. Dwivedi YK, Rana NP, Tamilmani K, Raman R. A meta-analysis based modified unified theory of acceptance and use of technology (meta-UTAUT): a review of emerging literature. Curr Opin Psychol. 2020;36:13-8.

14. McEvoy R, Ballini L, Maltoni S, O'Donnell CA, Mair FS, Macfarlane A. A qualitative systematic review of studies using the normalization process theory to research implementation processes. Implement Sci. 2014;9:2.

15. Lankton NK, Wilson EV, Mao E. Antecedents and determinants of information technology habit. Information \& Management. 2010;47(5):300-7.

16. Wilmer $\mathrm{HH}$, Chein JM. Mobile technology habits: patterns of association among device usage, intertemporal preference, impulse control, and reward sensitivity. Psychon Bull Rev. 2016;23(5):1607-14.

17. MacCoun RJ. The burden of social proof: shared thresholds and social influence. Psychol Rev. 2012;119(2):345-72.

18. Sah S, Fugh-Berman A. Physicians under the influence: social psychology and industry marketing strategies. J Law Med Ethics. 2013;41(3):665-72.

19. Amblee N, Bui T. Harnessing the Influence of Social Proof in Online Shopping: The Effect of Electronic Word of Mouth on Sales of Digital Microproducts. International Journal of Electronic Commerce. 2011;16(2):91-114.

20. Kuek A, Hakkennes S. Healthcare staff digital literacy levels and their attitudes towards information systems. Health Informatics J. 2020;26(1):592-612.

21. Ward R, Stevens C, Brentnall P, Briddon J. The attitudes of health care staff to information technology: a comprehensive review of the research literature. Health Info Libr J. 2008;25(2):81-97.

22. Rummery K, Coleman A. Primary health and social care services in the UK: progress towards partnership? Soc Sci Med. 2003;56(8):1773-82. 
23. Devlin AM, McGee-Lennon M, O'Donnell CA, Bouamrane MM, Agbakoba R, O'Connor S, et al. Delivering digital health and well-being at scale: lessons learned during the implementation of the dallas program in the United Kingdom. J Am Med Inform Assoc. 2016;23(1):48-59.

24. Digital N. Narrowing of NHS Gender divide but men still the majority in senior roles, 2018 [Available from: https://digital.nhs.uk/news-and-events/latestnews/narrowing-of-nhs-gender-divide-but-men-still-the-majority-in-senior-roles.

25. Gesulga JM, Berjame A, Moquiala KS, Galido A. Barriers to Electronic Health Record System Implementation and Information Systems Resources: A Structured Review. Procedia Computer Science. 2017;124:544-51.

26. Health Research Authority. Defining Research. NRES guidance to help you decide if your project requires review by a Research Ethics Committee [Available from: https://www.clahrc-eoe.nihr.ac.uk/wp-content/uploads/2014/04/defining-research.pdf.

\section{Figures}

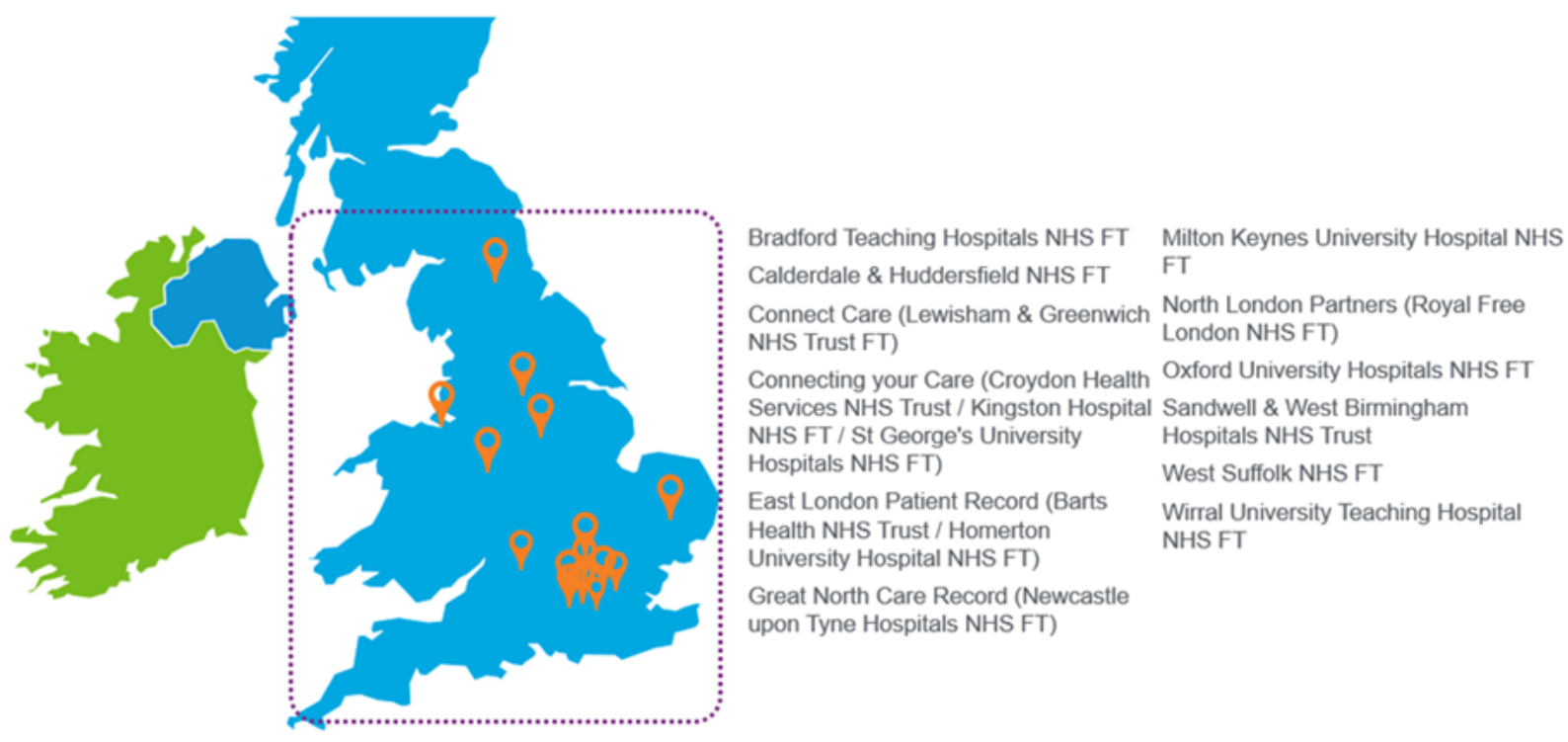

Figure 1

NHS Trusts in England utilising Cerner's HIE platform 